\title{
REVISED ALLOCATION OF A MEINERT SPECIES, WITH PROPOSAL OF A NEW SPECIES OF EURYTION (CHILOPODA : GEOPHILOMORPHA : CHILENOPHILIDAE)
}

\author{
By R. E. Crabill, Jr. \\ Smithsonian Institution, \\ U. S. National Museum, Washington, D. C. ${ }^{1}$
}

When Meinert described Geophilus tenebrosus as new in $1886^{2}$, he characterized it as having no ventral pores ("Pori ventrali nulli"), which almost surely accounts for Attem's erroneous referral of it to Brachygeophilus in his celebrated ordinal monograph of $1929 .{ }^{3}$ Meinert's original description is generally ambiguous; it provides no convincing clues that might have suggested to Attems that the species is an Eurytion.

The true generic identity of the Meinert form was readily apparent, when I examined the syntypical series in Copenhagen in I960. The specimens are plainly referable to Eurytion, a genus whose species are prevalent in southern South America and southern Africa.

Subsequently in a collection of Uruguayan specimens sent me for study of Dr. Pablo R. San Martin I discovered a specimen which is clearly referable to tenebrosum. This specimen, which agrees in all significant particulars with the syntypical material, is described here. The Uruguayan form was collected at Santa Clara de Olimar on November 17, 1958, by L. Lolessi. Because the origin of Meinert's material is unclear from his original description, I present here a detailed citation: Argentina, State of Gran Chaco, Riacho del Oro, $27^{\prime} \mathrm{O}^{\prime \prime} \mathrm{S}, 58^{\prime} 33^{\prime \prime} \mathrm{W}$.

Meinert's syntypical series is comprised of five specimens: two females each with 57 leg pairs; two females each with 59 leg pairs; one male with 55 leg pairs. Of these a female with $57 \mathrm{leg}$ pairs has been selected by me as the lectotype and appropriately labelled as such in the Copenhagan collection.

\footnotetext{
${ }^{1}$ This study was undertaken with the aid of a grant from the National Science Foundation. I should also like to express my gratitude for the loan of specimens and their hospitality to: Dr. Herbert W. Levi, Museum of Comparative Zoology, Harvard; Dr. S. L. Tuxen, Universitetets Zoologiske Museum, Copenhagen; Dr. Pablo R. San Martin, Museo Nacional de Historia Natural, Montevideo.

${ }^{2}$ Vidensk. Meddel., vols. 36-38, p. 146.

${ }^{3}$ Das Tierreich, Lief, 52, p. 192.
} 
Eurytion tenebrosum ${ }^{4}$ differs from all known South American members of the genus in at least two regards: the second maxillary coxosternum bears a pair of lappets; the second maxillary circumforaminal ring, which is notably elongate, is bordered by a vague hyaline suture.

\section{Eurytion tenebrosum (Meinert)}

GENERAL. Length, $40 \mathrm{~mm}$. Leg pairs, 55. Color, brownish yellow. Shape: last io segments slightly attenuate; anteriorly not so. antennae. Filiform, not flattened. First 7 articles sparsely clothed with long setae; 8-I 4 densely, shortly setose. CEPHALIC PLATE. Much longer than wide, 24 : 19. Frontal suture absent. CLYPEUS. Much wider than long. Fenestra minutely punctate, not areolate, with I-2 inclusive setae. Setae: prefenestrals absent, parafenestrals $I-I$, intrafenestrals $I-2$, postfenestrals and midclypeals ca. 6-6. Plagulae absent. LABRUM. Small subtriangular midpiece completely separating sidepieces. FIRST MAXILlaE. Telopodite lappets slightly longer than telopodite. Coxosternal lappets present but short. SECOND MAXILLAE. Isthmus shallow, narrow, hyaline, not areolate. Mesodistal process absent. Pore surrounded by sclerotized rim. Telopodite: basal condyles both present, equal; articles without denticles; claw long, simple, curved.

PREHENSOR. Flexed, projecting well beyond head margin. Tarsungular edge smooth, not serrulate. Denticles: article 4 with prominent basal tooth; articles 2 and 3 unarmed; article $I$ with two denticles, the distal larger than the proximal. PRosternum. Anterior denticles yellowish. Pleuroprosternal sutures parallel to sides of prosternum. Pleurograms absent. LEGS. Without dense vestiture; articles 4 and 5 each with 3 exceptionally long setae. Parungues: anteriors very short, about $\mathrm{I} / 4$ as long as claws; posteriors minute, nearly suppressed. STERnites. Porefields on 2 through penult; each single, subcircular on anterior body third, thereafter double and assuming more posterior positions.

ultimate Pedal segment. Pretergite laterally non-fissate, fused with its pleurites. Presternite midlongitudinally weakly suturate. Sternite: sides slightly excurved, rear straight. Each coxopleuron with three gland complexes, each heterogenous in the form of a vague rosette. Telopodite: very slightly crassate, about I/4 longer

${ }^{4}$ The gender of the generic name has been expressed variously; some have considered it neuter, some masculine. That Eurytion is the name of a male mythological figure does not alter its being a neuter Greek substantive. 

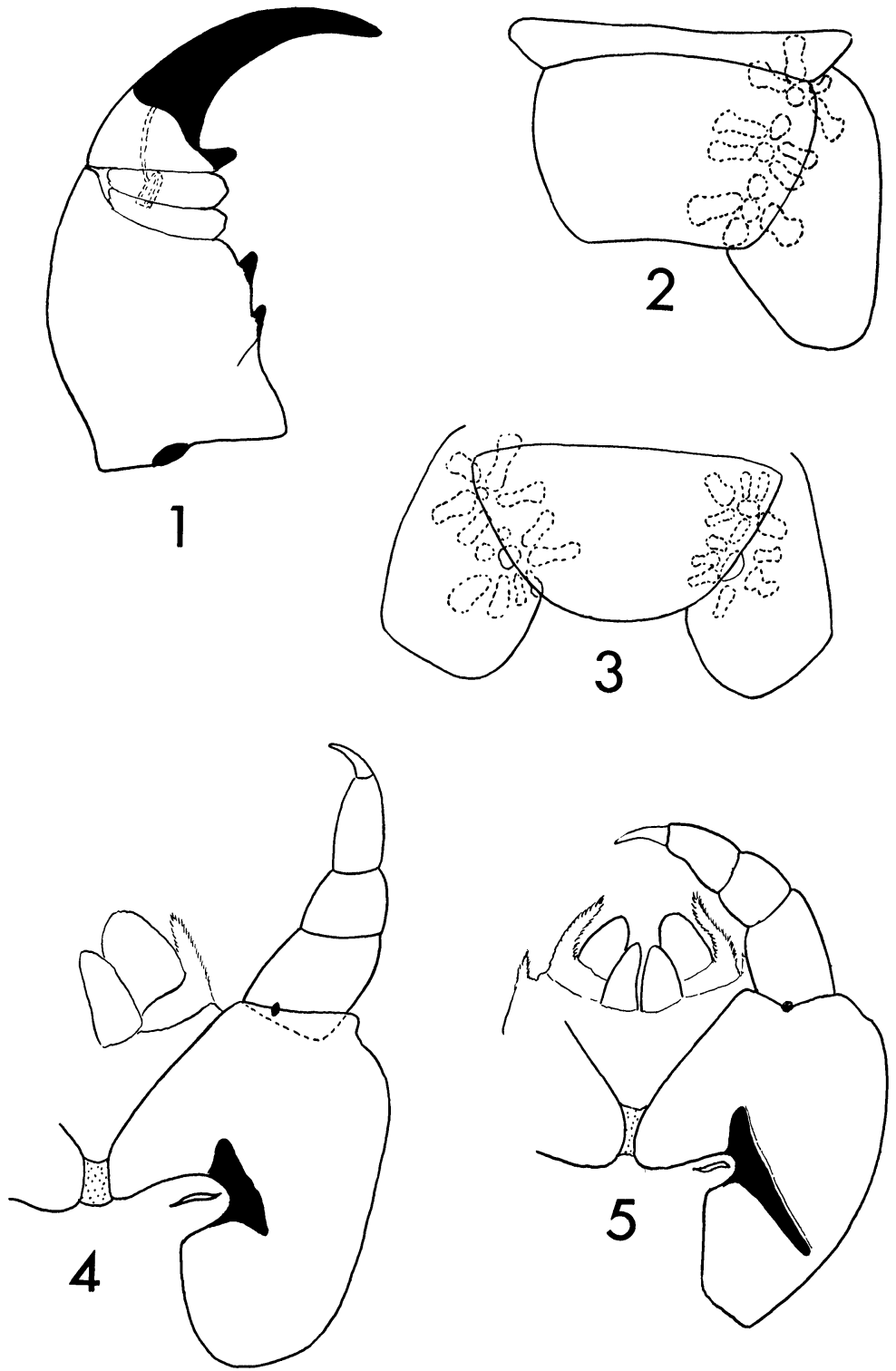

Crabill - Eurytion 
than penult; tarsalia 2, narrow than preceding articles; pretarsus strongly unguiform.

POSTPEDAL SEGMENTs. Female gonopods medially fused, uniarticular. Anal pores present.

The new species described below differs from all known members of the genus in that each coxopleuron possesses two rosettes of gland canals. Eurytion lethifer seems most like E. mundum (Chamberlin), from Chile, which was initially proposed as the type-species of a new genus, Chilerium $^{5}$, here considered to be a junior subjective synonym of Eurytion Attems (New Synonym). In E. mundum each coxopleuron has only one rosette of gland canals.

\section{Eurytion lethifer, new species}

Holotype: female. Peru: Cuzco, Urubamba at $2880 \mathrm{~m}$. February I8, 1965. Herbert W. Levi, leg. Deposited in the Museum of Comparative Zoology, Harvard.

GENERAL. Length, $35 \mathrm{~mm}$. Leg pairs, 59. Color, brownish yellow. Shape: last ro segments slightly attenuate; anteriorly not so. antennae. Filiform, not flattened. First 7 articles sparsely clothed with long setae; 8-I 4 densely clothed with short setae. CEPHALIC PLATE. Much longer than wide, 2I : I5. Cephalic suture absent. CLYPEUS. Much wider than long. Fenestra minutely punctate, not areolate, with 2 inclusive setae. Plagulae absent. Setae: 2 prefenestrals, 2 intrafenestrals, I postfenestral, 2 midclypeals. LABRUM. Sidepieces very narrowly separated by triangular midpiece. FIRST maxillae. Telopodite lappets scabrous, long. Coxosternal lappets absent. SECOND MAXILLAE. Isthmus shallow, narrow, medially hyaline, not areolate. Mesodistal process absent. Pore surrounded by sclerotized rim. Telopodite: basal condyles both present, equal; articles without denticles; claw long, simple, curved.

PREHENSOR. Flexed, projecting well beyond head margin. Tarsungular edge smooth, not serrulate. Denticles: article 4 with prominent basal tooth; articles 2 and 3 unarmed; article $I$ with two

${ }^{5}$ Lunds Universitets Arrskrift, N.F. Avd. 2, Bd. 51(5), p. 23, 1955.

Explanation of Plate 16

Eurytion tenebrosum (Meinert): 2, 5. Eurytion lethifer, sp. n. : 1, 3, 4.

1. Right prehensor; ventral aspect. 2. Ultimate pedal segment; ventral aspect. 3. Ultimate pedal segment; ventral aspect. 4. First and second maxillae; ventral aspect. 5. First and second maxillae; ventral aspect. 
pigmented denticles, the distal larger than the proximal. PROSTERNUM. With 2 prominent anterior denticles, these yellowish, not brown. Pleurograms absent. Pleuroprosternal sutures strictly parallel to sides of prosternum. LEGS. Without dense vestiture; articles 3, 4, 5 , and 6 each ventrally with one especially long seta. Parungues: anteriors nearly as long as claws; posteriors minute, nearly suppressed. STERnites. Porefields on 2 through penult; each single, transversely slightly elliptical, postcentral on anterior body third, thereafter dividing in two and assuming more posterior positions.

Ultimate Pedal Segment. Pretergite laterally non-fissate, fused with its pleurites. Presternite midlongitudinally weakly suturate. Sternite posteriorly broadly rounded. Coxopleural glands: 2 per coxopleuron, each heterogeneous, their canal disposed in rough rosettes about each exit pore. Telopodite: very slightly crassate, a quarter longer than penult; tarsalia 2, narrower than proximal articles; pretarsus unguiform, long.

POSTPEDAL SEGMENTS. Female gonopods medially fused, uniarticular. Anal pores absent. 

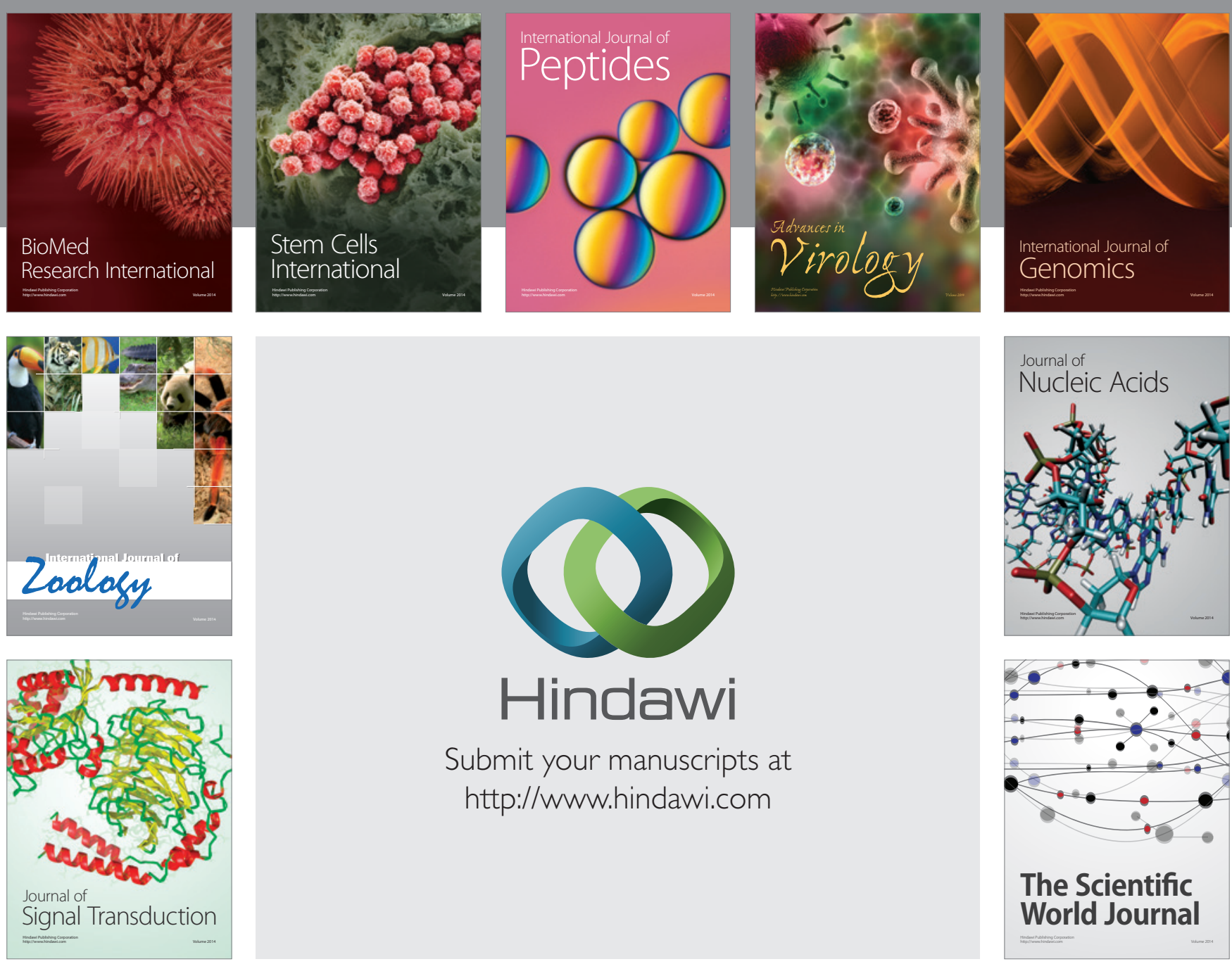

Submit your manuscripts at

http://www.hindawi.com
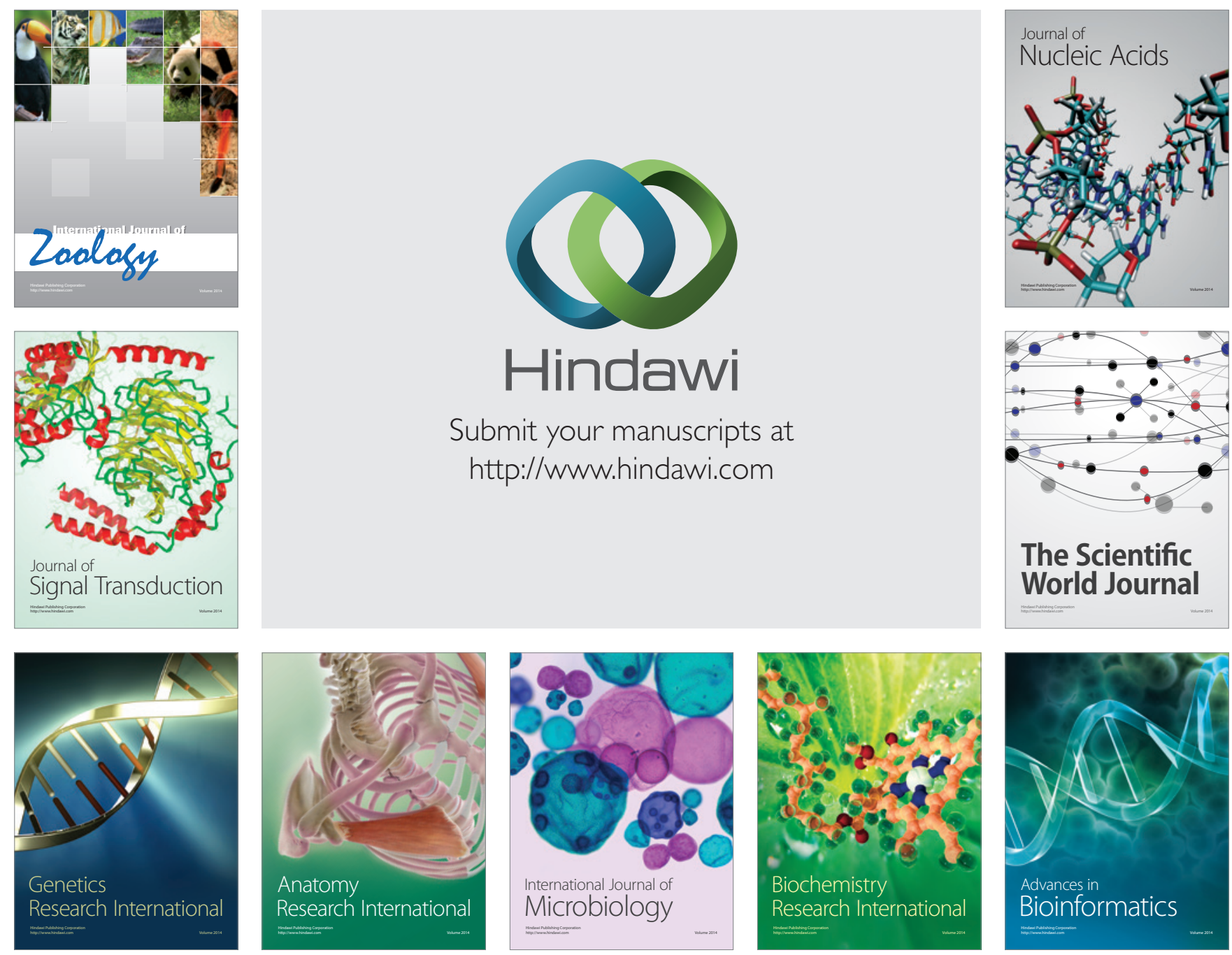

The Scientific World Journal
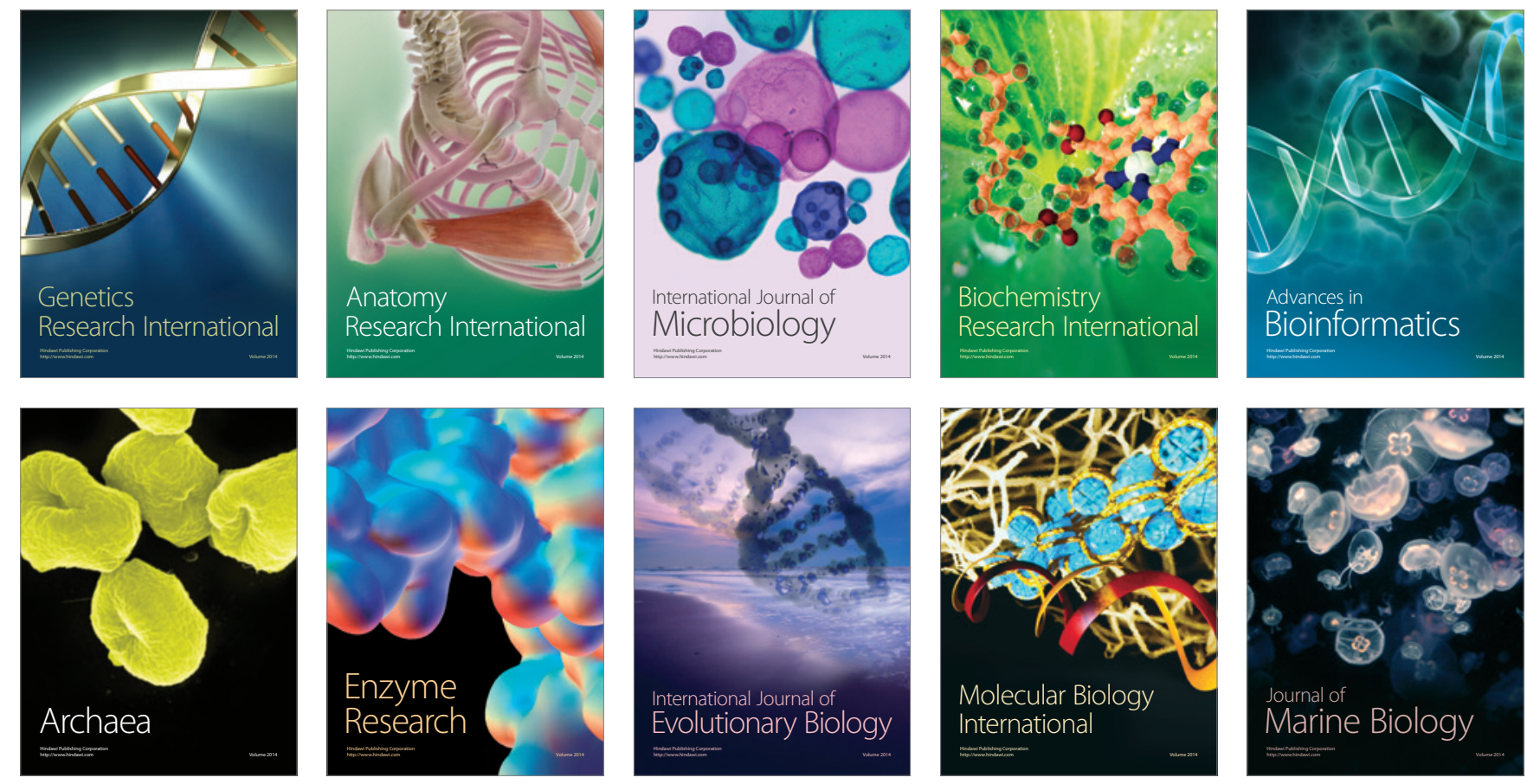